Periodica Polytechnica Transportation Engineering, 49(3), pp. 242-249, 2021

\title{
Vehicle Semi-active Suspension Control with Cloud-based Road Information
}

\author{
Hakan Basargan, András Mihály², Ádám Kisari², Péter Gáspár², Olivier Sename³ \\ 1 Department of Control for Transportation and Vehicle Systems, Faculty of Transportation and Vehicle Engineering, Budapest \\ University of Technology and Economics, H-1111 Budapest, 2 Stoczek street, Hungary \\ 2 Systems and Control Laboratory, Institute for Computer Science and Control (SZTAKI), Eötvös Loránd Research Network (ELKH) \\ H-1111 Budapest, 13-17 Kende street, Hungary \\ ${ }^{3}$ GIPSA-lab, INPG, Université Grenoble Alpes, 38402 Grenoble, 11 Rue des Mathématiques, France \\ * Corresponding author, e-mail: hakan.basargan@kjk.bme.hu
}

Received: 18 May 2021, Accepted: 15 June 2021, Published online: 04 August 2021

\begin{abstract}
Adaptive suspension control considering passenger comfort and stability of the vehicle has been researched intensively, thus several automotive companies already apply these technologies in their high-end models. Most of these systems react to the instantaneous effects of road irregularities, however, some expensive camera-based systems adapting the suspension in coherence with upcoming road conditions have already been introduced. Thereby, using oncoming road information the performance of adaptive suspension systems can be enhanced significantly. The emerging technology of cloud computing enables several promising features for road vehicles, one of which may be the implementation of an adaptive semi-active suspension system using historic road information gathered in the cloud database. The main novelty of the paper is the developed semi-active suspension control method in which Vehicle-to-Cloud-to-Vehicle technology serves as the basis for the road adaptation capabilities of the suspension system. The semiactive suspension control is founded on the Linear Parameter-Varying framework. The operation of the presented system is validated by a real data simulation in TruckSim simulation environment.
\end{abstract}

Keywords

cloud-based control, cloud computing, semi-active suspension control, adaptive semi-active suspension control, LPV control

\section{Introduction}

Cloud computing has turned out to be a key topic along with the development of network systems. It is a significant technology for the application of optimization computations and advanced vehicle control. The vehicles, which have advanced technology consist of hundreds of embedded electronic control units (ECU). The substantial onboard computing power and storage is necessary due to advanced algorithm usages. But computing power and storage is not cost-effective, thus cloud computing is a solution for the huge algorithm load and database complexity.

Suspension system design has a significant role in vehicle technology. A well-designed suspension system radically improves both road holding and driving comfort. Estimation of the road condition is necessary to design an adaptive semi-active suspension system. Reconfiguration of the suspension system is possible at different road irregularities. This reconfiguration finds a

trade-off between driving comfort and road holding. This reconfiguration is based on the road condition and performance importance of the vehicle. The cloud technology enables the receiving of road conditions and information for the suspension system.

The suspension control from the perspective of Vehicleto-Cloud-to-Vehicle(V2C2V) methods have been studied recently, (Guanetti and Borelli, 2017; Li et al., 2014; Li et al., 2015; Mihaly et al., 2017; Zheng et al., 2019).

This paper presents a novel method of a cloudbased adaptive semi-active suspension control system. The reconfigurable semi-active suspension system has been developed by using $\mathrm{V} 2 \mathrm{C} 2 \mathrm{~V}$ technology, where adaptation of the vehicle for the road irregularities is possible. The advantage of the proposed method is the adaptation to the road conditions without using hardware-related technology, which may cost more. 
Several researchers have already developed semi-active suspension systems based on the quarter-car model with very different approaches. A skyhook control strategy has been widely used for the semi-active suspension control (Liu et al., 2019; Moaaz and Ghazaly, 2019; Shimoya and Katsuyama, 2019), where the strategy is based on designing the semi-active suspension control so that the chassis is linked to the sky to reduce the vertical accelerations of the chassis and of the axle independently of each other. It is easy to implement, however it disrupts the dynamic tire load. The model predictive control (MPC) (Rathai et al., 2019a; Rathai et al., 2019b) method is also widely used but it lacks robustness properties. Unlike previous approaches, control method (Ye and Zheng, 2019; Yu and Zhang, 2019) guarantees ride comfort, road holding and vehicle stability performances, however dynamic control reconfiguration is not possible due to the fixed weighting of the performances. Due to reconfiguration importance in the semi-active suspension control, the control method, which has availability for realtime reconfiguration has to be considered. Thus, the control is founded on the Linear Parameter-Varying(LPV) framework in this paper due to its availability for the dynamic configuration by modifying the scheduling variable, see (Basargan et al., 2021; Basargan et al., 2020a; Basargan et al., 2020b; Morato et al., 2019; Pham, 2020).

The paper is organized as follows: Section 2 presents the control-oriented quarter-car model and the LPV controller synthesis. Section 3 introduces the architecture of the cloud application and the designing of the scheduling variable. Section 4 demonstrates the operation of the proposed method in a high-fidelity TruckSim simulation environment with real road data. Finally, concluding remarks are presented in Section 5.

\section{Modeling and control synthesis of the suspension system}

This section contains the modeling of the quarter-car suspension and designing the reconfigurable semi-active suspension control through LPV method.

The layout of the commonly used two-degree-of-freedom quarter-car model is shown in Fig. 1. It is widely used due to its controllability and simplicity. The dynamic model of the quarter-car suspension system and the actuator is written as Eqs. (1) and (2).

$$
\begin{aligned}
& m_{s} \ddot{q}_{1}+b_{s}\left(\dot{q}_{1}-\dot{q}_{2}\right)+k_{s}\left(q_{1}-q_{2}\right)+F_{m r}=0 \\
& m_{u} \ddot{q}_{2}+b_{s}\left(\dot{q}_{2}-\dot{q}_{1}\right)+k_{t}\left(q_{2}-w\right) \\
& +k_{s}\left(q_{2}-q_{1}\right)-F_{m r}=0 .
\end{aligned}
$$

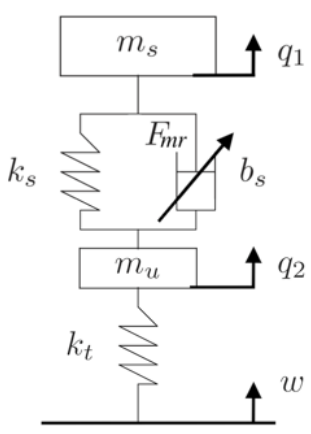

Fig. 1 Quarter-car model

$\dot{F}_{m r}=-\frac{1}{\tau} F_{m r}+\frac{1}{\tau} u$

Here, $m_{s}$ is the sprung mass of the quarter vehicle, $m_{u}$ represents the unsprung mass, $k_{t}$ is the stiffness of the tire, $k_{s}$ is the stiffness of the spring, $b_{s}$ is the damping rate of the shock absorber, while $F_{m r}$ is the control force generated by the actuator and $u$ is the control input of the system.

Note, that $q_{1}$ and $q_{2}$ are the vertical displacement of the sprung mass and the unsprung mass, while road disturbance is denoted with $w$. The nominal parameters of the quarter-car model for the front and rear suspension is shown in Table 1.

The unmodeled dynamics $\Delta$ are also taken into consideration with $\left|\Delta\left(w_{1}\right)\right|=25$ at low frequencies and $\left|\Delta\left(w_{2}\right)\right|=1$ at high frequencies, and is assumed to be stable with the norm condition $\|\Delta\|_{\infty}<1$.

The state vector is selected as with the below elements:

$$
\begin{aligned}
& x_{1}=q_{1} \\
& x_{2}=q_{2} \\
& x_{3}=\dot{q}_{1} \\
& x_{4}=\dot{q}_{2} \\
& x_{5}=F_{m r},
\end{aligned}
$$

Next, performance specifications are defined in order to achieve a desired trade-off between ride comfort and road holding, while also taking control force into consideration:

- Hence, for the sake of increasing passenger comfort, the acceleration of the sprung mass must be minimized with the following optimization criterion: $z_{1}=\dot{q}_{1} \rightarrow 0$.

Table 1 Parameters of the suspension

\begin{tabular}{lccc}
\hline Parameters (symbols) & $\begin{array}{c}\text { Front } \\
\text { suspension }\end{array}$ & $\begin{array}{c}\text { Rear } \\
\text { suspension }\end{array}$ & Unit \\
\hline Suspension stiffness $\left(k_{s}\right)$ & 30 & 60 & $\mathrm{kN} / \mathrm{m}$ \\
Tire stiffness $\left(k_{t}\right)$ & 220 & 220 & $\mathrm{kN} / \mathrm{m}$ \\
Sprung mass $\left(m_{s}\right)$ & 214 & 336 & $\mathrm{~kg}$ \\
Unsprung mass $\left(m_{u}\right)$ & 40 & 40 & $\mathrm{~kg}$ \\
Time constant $(\tau)$ & $1 / 30$ & $1 / 30$ & $\mathrm{~s}$ \\
Damping $\left(b_{s}\right)$ & 50 & 50 & $\mathrm{~N} / \mathrm{m} / \mathrm{s}$ \\
\hline
\end{tabular}


- Directional stability is guaranteed with the minimization of suspension deflection, hence $z_{2}=\left(q_{1}-q_{2}\right) \rightarrow 0$.

- In order to reduce variations of side force to guarantee stability, the dynamic tire load must be minimized with the optimization criterion: $z_{3}=\left(q_{2}-w\right) \rightarrow 0$.

- Finally, in order to avoid actuator saturation, the control force must also be considered with the optimization criterion $z_{4}=F_{m r} \rightarrow 0$.

These performances are put in a performance vector $\mathbf{z}=\left[z_{1} z_{2} z_{3} z_{4}\right]$. The measured signal is the relative displacement between the masses, i.e $x_{1}-x_{2}$ The control input $u=F_{m r}$ is the vertical force generated by the actuator, with the dynamics listed in Eq. (2).

Next, the system is given with Eq. (1) needs to be transformed into the state-space representation form as Eq. (4).

$\dot{\mathbf{x}}=A_{x}+B_{1} w+B_{2} u$

$\mathbf{z}=C_{1} x+D_{11} w+D_{12} u$

$\mathbf{y}=C_{2} x+D_{21} w+D_{22} u$.

The proposed high-level controller is founded on a weighting strategy formulated through a closed-loop architecture shown in Fig. 2.

Here, $G$ is the quarter-car control-oriented model defined in Eq. (4). $K$ is the designed LPV controller characterized with the scheduling variable $\rho$ responsible for control reconfiguration, $u$ is the control input of the actuator, $y$ is the measured output, $n$ is the measurement noise, $z$ represents the performance outputs and $w$ stands for the road disturbance.

The uncertainties of the quarter-car model are considered with weighting function $W_{r}$ and $\Delta$. Note, that weighting functions should be considered as penalty functions, hence weights should be large where small signals are desired and vice versa. The aim of weighting function $W_{n}$ is to represent sensor noises, while weighting function $W_{w}$

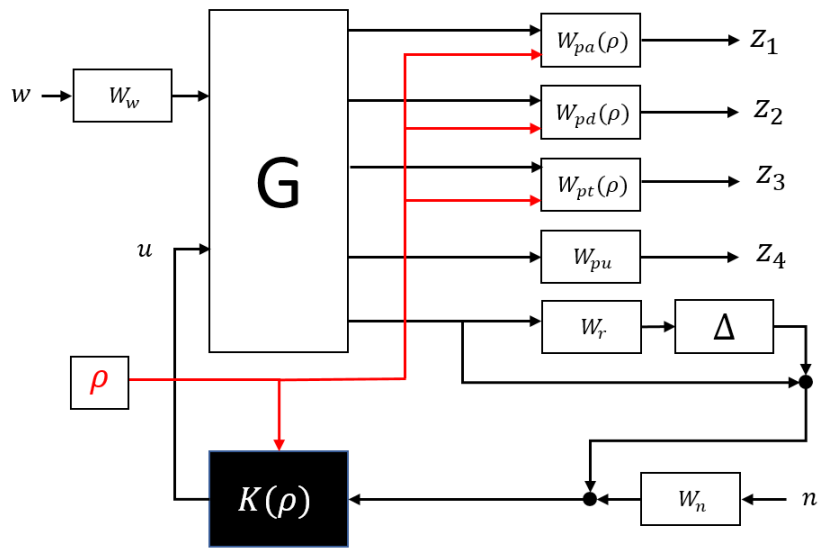

Fig. 2 Closed-loop interconnection stands for the road disturbances. The goals of weighting functions given in $W_{p}$ are to keep the sprung mass acceleration $\left(W_{p a}\right)$, suspension deflection $\left(W_{p d}\right)$, tire deflection $\left(W_{p t}\right)$ and control input $\left(W_{p u}\right)$ small over the required frequency range. Note, that while $W_{p a}$ represents passenger comfort, $W_{p d}$ and $W_{p t}$ stands for directional stability and dynamic tire load. Since the predefined performance specifications may conflict with each other, weighting functions given in $W_{p}$ must be designed in a manner that an appropriate trade-off can be guaranteed between them. Moreover, in order to ensure control reconfiguration in case one of the predefined performances becomes more important due to estimated future road conditions, a scheduling variable $\rho \in[0,1]$ is introduced to shape weighting functions $W_{p a}$, $W_{p d}$ and $W_{p t}$. Hence, these performance weighting functions related to passenger comfort and road holding are selected in a second-order proportional form as follows:

$$
\begin{aligned}
& W_{p t}=(1-\rho) \frac{\alpha_{3} s+1}{T_{3} s+1} \\
& W_{p d}=(1-\rho) \frac{\alpha_{2} s+1}{T_{2} s+1} \\
& W_{p a}=\rho \frac{\alpha_{1} s+1}{T_{1} s+1},
\end{aligned}
$$

where $\alpha_{1,2,3}$ and $T_{1,2,3}$ are designed parameters. Note, that weighting functions $W_{p u}, W_{r}, W_{n}$ and $W_{w}$ are all given in similar linear and proportional form without containing the scheduling variable $\rho$.

The LPV performance problem is to choose a parameter-varying controller, which guarantees quadratic stability for the closed-loop system while the induced $L_{2}$ norm from the disturbance $w$ to the performances $z$ is smaller than the value $\gamma$, as described in (Bokor and Balas, 2005). Hence, the minimization task is given as:

$$
\inf _{K \varrho \in F_{p} w_{2} \neq 0, w \in \mathcal{L}_{2}} \frac{\|z\|_{2}}{\|w\|_{2}} \leq \gamma .
$$

The solution of an LPV problem is governed by the set of infinite dimensional LMIs being satisfied for all $\rho \in F_{p}$ thus it is a convex problem. In practice, this problem is set up by gridding the parameter space and solving the set of LMIs that hold on the subset of $F_{p}$, see (Wu et al., 1995).

The result of the presented design is a reconfigurable LPV controller, where $\rho=1$ stands for a setup where passenger comfort is preferred, whereas $\rho=0$ represents a controller focusing on stability and road holding of the vehicle. When $\rho$ is between these edge values, a combination of performances are guaranteed by the controller. 
The magnetorheological (MR) damper has a limitation on the control input force, thus a semi-optimal solution is given in Eq. (7).

$$
F_{m r}= \begin{cases}F_{o p t}, & \text { if } \dot{q}_{1}\left(\dot{q}_{1}-\dot{q}_{2}\right)>0 \\ F_{p a s}, & \text { if } \dot{q}_{1}\left(\dot{q}_{1}-\dot{q}_{2}\right)<0\end{cases}
$$

Here, $F_{o p t}$ and $F_{p a s}$ are optimal and passive suspension forces and $\dot{q}_{1}-\dot{q}_{2}$ is the deflection velocity of the suspension.

\section{System integration and decision algorithm}

This section consists of the architecture of the system, integration of the cloud application and designing of the scheduling variable.

\subsection{Architecture of the system}

In this research the mentioned capabilities of the network and computing technologies are used to improve the safety and comfort of such equipped road vehicles. The whole system consists of the following: the vehicle is equipped with semi-active suspension system, controlled by the LPV controller obtained in the previous section; the cloud system communicates with the vehicle and gathers, processes, stores and distributes the data. The architecture of the proposed system is depicted in Fig. 3.

The process is the following:

- measurement data is collected by the on-board unit from the suspension and uploaded to the cloud,

- the road-information database is built by gathering information from vehicles by the cloud application,

- performance index defines the importance of the performance, between road holding and driving comfort in the cloud application,

- based on the performance index, road irregularity, vehicle velocity and performance results, the corresponding scheduling variable is calculated by the decision algorithm in the cloud.

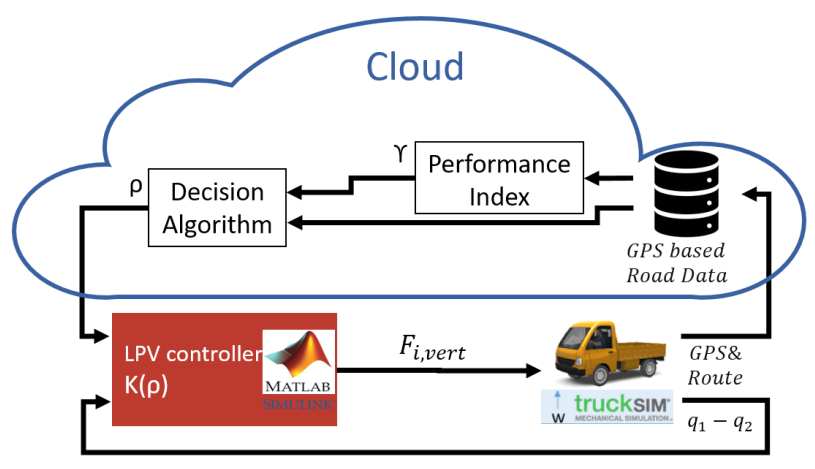

Fig. 3 Architecture of the system
The cloud part of the system is implemented on a private infrastructure cloud, while the introduced architecture could be implemented on the other clouds without major modifications. MongoDB database engine has been selected due to its features, where handling geospatial information is possible. The cloud application is written in Python and it uses the Flask framework to provide a RESTful API to the cloud functionality.

As it can be seen in Fig. 3, the vehicle is simulated for this paper using TruckSim software. The simulation environment is integrated with Matlab, where controller test and connection to the cloud via web API is possible.

The system performs two steps in order to choose the corresponding scheduling variable. In the first step, the performance index is defined in the cloud application, while the decision algorithm defines the $\rho$ in the second step.

The on-board computer collects the current velocity, position of the vehicle, deformation of the tire, vertical acceleration of the body, and displacement between sprung and unsprung masses. All these collected parameters are forwarded to the cloud application via a mobile internet connection.

The performance index is calculated based on previous measurements, which are stored in the cloud database. According to vertical acceleration and tire deformation values, the importance of one of these performances is selected. When new data arrives, the cloud application calculates the scheduling variable for the LPV controller. This calculation is based on the performance index. The calculated scheduling variable is sent to the semi-active suspension LPV controller in order to calculate corresponding damping forces.

\subsection{Design of scheduling variable}

Defining the performance index is significant to find the importance of the two performances. According to the velocity of the vehicle and performance results, we need to define which performance is more important than the other one. Thus, normalized vertical acceleration and tire deformation values are needed to be calculated for the comparison.

The cloud-based road database has multiple measurements and historical data with different road irregularities and velocities. These data and measurements are the type of road irregularity, location of road irregularities, the velocity of the vehicle, deformation value of the tire, acceleration of the body, the displacement between sprung and unsprung masses at each time step. It is necessary to categorize and group all these data in order to compare them 
with the data which arrives from the on-board computer. Thus, root-means-square (RMS) of vertical body acceleration and tire deformation has been calculated with Eq. (8):

$$
\begin{aligned}
R^{v a} & =\sqrt{\frac{1}{T_{a}} \int_{0}^{T_{a}} a^{2}(t) d t} \\
R^{t d} & =\sqrt{\frac{1}{T_{t}} \int_{0}^{T_{t}} t_{d}^{2}(t) d t},
\end{aligned}
$$

where, $R^{v a}$ and $R^{t d}$ are RMS values of vertical acceleration of body and deformation of the tire, $t_{d}(t)$ and $a(t)$ are the time-weighted tire deformation and vertical acceleration, $T_{a}$ and $T_{t}$ is the number of acceleration and tire deformation values. Their normalized value is calculated as follow in Eq. (9):

$$
\zeta_{i, j}^{k}=\frac{100 \mathcal{R}_{i, j}^{k}}{\mathcal{R}_{\max }^{k}},
$$

where, $\zeta$ is the normalized value, $k \in[v a, t d]$ is performance type, where $v a$ is the vertical acceleration performance, $t d$ is the tire deformation performance, $i$ is the road type and $j$ is the velocity of the vehicle. In the case of the velocity of the simulated vehicle which differs from the cloud, the interpolation method is used to find dedicated performance values for different velocities and irregularities. Table 2 shows a couple of road data with different velocities and road irregularities and their RMS value of performances and normalized values in the cloud. This cloud data is built by gathering information from vehicles, which have passive suspension (Basargan et al., 2021).

The first step is finding the performance index $(\Upsilon)$, where it is possible to define the importance between two performances. The solution is provided by comparing their normalized values. The performance index $\Upsilon=0$ stands for tire deformation consideration (road holding), $\Upsilon=1$ stands for the vertical acceleration consideration (driving comfort) and $\Upsilon=0.5$ expresses that both performance considerations should be equal. Here, consideration means that, according to performance consideration, the designed scheduling variable should be close to zero or one. In the case of vertical acceleration consideration, the scheduling variable needs to be close to one, while in the tire deformation consideration scheduling variable needs to be close to zero. Equation (10) describes selection of the performance index. This index is calculated in the performance index block:

$$
\Upsilon= \begin{cases}0, & \zeta_{i, j}^{t d}>\zeta_{i, j}^{v a} \\ 1, & \zeta_{i, j}^{d d}<\zeta_{i, j}^{v a} . \\ 0.5, & \zeta_{i, j}^{t d}=\zeta_{i, j}^{v a}\end{cases}
$$

\begin{tabular}{|c|c|c|c|c|c|}
\hline $\begin{array}{l}\text { Road } \\
\text { irregularity }\end{array}$ & Velocity $(\mathrm{km} / \mathrm{h})$ & $R^{v a}$ & $\zeta^{v a}$ & $R^{t d}$ & $\zeta^{t d}$ \\
\hline \multirow{4}{*}{$5 \mathrm{~cm}$ bump } & 20 & 0.058 & 4.39 & 0.0005 & 4.54 \\
\hline & 40 & 0.115 & 8.71 & 0.0012 & 10.9 \\
\hline & 60 & 0.217 & 16.41 & 0.0022 & 20 \\
\hline & 80 & 0.293 & 22.15 & 0.0033 & 30 \\
\hline \multirow{4}{*}{$5 \mathrm{~cm}$ pothole } & 20 & 0.0581 & 4.38 & 0.0005 & 4.54 \\
\hline & 40 & 0.1165 & 8.78 & 0.0013 & 11.82 \\
\hline & 60 & 0.2178 & 16.42 & 0.0022 & 20 \\
\hline & 80 & 0.290 & 21.87 & 0.0032 & 29 \\
\hline \multirow{4}{*}{$7 \mathrm{~cm}$ bump } & 20 & 0.081 & 6.11 & 0.0005 & 4.54 \\
\hline & 40 & 0.160 & 12.06 & 0.0013 & 11.8 \\
\hline & 60 & 0.291 & 21.94 & 0.0022 & 20 \\
\hline & 80 & 0.401 & 30.24 & 0.0033 & 30 \\
\hline \multirow{4}{*}{$7 \mathrm{~cm}$ pothole } & 20 & 0.081 & 6.1 & 0.0005 & 4.54 \\
\hline & 40 & 0.1625 & 12.25 & 0.0013 & 11.8 \\
\hline & 60 & 0.3091 & 21.31 & 0.0022 & 20 \\
\hline & 80 & 0.4784 & 36.07 & 0.0033 & 30 \\
\hline \multirow{4}{*}{$10 \mathrm{~cm}$ bump } & 20 & 0.115 & 8.71 & 0.0013 & 11.8 \\
\hline & 40 & 0.217 & 16.4 & 0.0022 & 20 \\
\hline & 60 & 0.293 & 22.1 & 0.0033 & 30 \\
\hline & 80 & 0.537 & 40.5 & 0.0036 & 35.4 \\
\hline \multirow{4}{*}{$\begin{array}{l}10 \mathrm{~cm} \\
\text { pothole }\end{array}$} & 20 & 0.115 & 8.672 & 0.0005 & 4.54 \\
\hline & 40 & 0.22 & 16.6 & 0.0014 & 12.72 \\
\hline & 60 & 0.5042 & 38.02 & 0.0067 & 60.9 \\
\hline & 80 & 0.9053 & 68.1 & 0.0081 & 73.3 \\
\hline \multirow{4}{*}{$\begin{array}{l}\text { Multiple } \\
\text { bumps and } \\
\text { potholes }\end{array}$} & 20 & 0.370 & 27.9 & 0.0110 & 100 \\
\hline & 40 & 0.431 & 32.5 & 0.0017 & 15.4 \\
\hline & 60 & 0.466 & 35.2 & 0.0029 & 26.3 \\
\hline & 80 & 0.444 & 33.5 & 0.0041 & 37.2 \\
\hline \multirow{4}{*}{ Sinusoidal } & 20 & 0.431 & 32.5 & 0.007 & 63.6 \\
\hline & 40 & 0.751 & 56.6 & 0.002 & 21.8 \\
\hline & 60 & 1.122 & 84.6 & 0.003 & 27.2 \\
\hline & 80 & 1.326 & 100 & 0.011 & 100 \\
\hline
\end{tabular}

Table 2 Cloud data

The second step calculates the scheduling variable by considering performance index and normalized performance values in the decision algorithm block. Equation (11) calculates the corresponding scheduling variable:

$\rho=\left\{\begin{array}{ll}1-\frac{\zeta_{i, j}^{t d}}{\zeta_{i, j}^{v a}+\zeta_{i, j}^{t d}}, & \Upsilon=0 \\ \frac{\zeta_{i, j}^{v a}}{\zeta_{i, j}^{v a}+\zeta_{i, j}^{t d}}, & \Upsilon=1 . \\ 0.5, & \Upsilon=0.5\end{array}\right.$.

The designed scheduling variable is forwarded to the LPV controller, where dedicated damping force is calculated. 


\section{Simulation results}

The vehicle selected for simulation is a compact utility truck having independent front and rear semi-active suspension.

The simulation route is based on a Hungarian highway road from Gyöngyös to Kápolna, which had been implemented in TruckSim environment based on real geographical data, having the speed limits and road distortions depicted in Table 3.

The altitude of the 3 kilometer long road section changes significantly while it contains three bigger curves as well at around $400 \mathrm{~m}, 1000 \mathrm{~m}$ and $1700 \mathrm{~m}$.

In order to demonstrate the effectiveness of the proposed method two different simulations have been performed and compared, one with the utility truck having conventional semi-active suspension and another where it was equipped with the proposed cloud-based adaptive semi-active suspension. The velocity profile of the simulated vehicles are depicted in Fig. 4.

The result of the cloud-based road consideration can be observed in Fig. 5, where the scheduling variable $\rho$ for the adaptive LPV controller and the nominal controller is depicted.

The performances of the conventional and the cloudbased adaptive method are compared next. It is well demonstrated in Fig. 6, that both vertical and lateral accelerations have been reduced at the road distortions with the proposed method, which greatly improves passenger comfort.

Table 3 Road irregularities and designed $\rho$

\begin{tabular}{lccc}
\hline Road irregularity & $\begin{array}{c}\text { Velocity } \\
(\mathrm{km} / \mathrm{h})\end{array}$ & $\begin{array}{c}\text { Location } \\
(\mathrm{m})\end{array}$ & Designed \\
\hline $\begin{array}{l}\text { Multiple bumps and } \\
\text { potholes }\end{array}$ & 70 & 150 & 0.5197 \\
$5 \mathrm{~cm}$ pothole & 55 & 550 & 0.447 \\
$7 \mathrm{~cm}$ pothole & 70 & 900 & 0.5459 \\
Sinusoidal & 70 & 1650 & 0.5992 \\
15 cm pothole & 80 & 2300 & 0.5336 \\
\hline
\end{tabular}

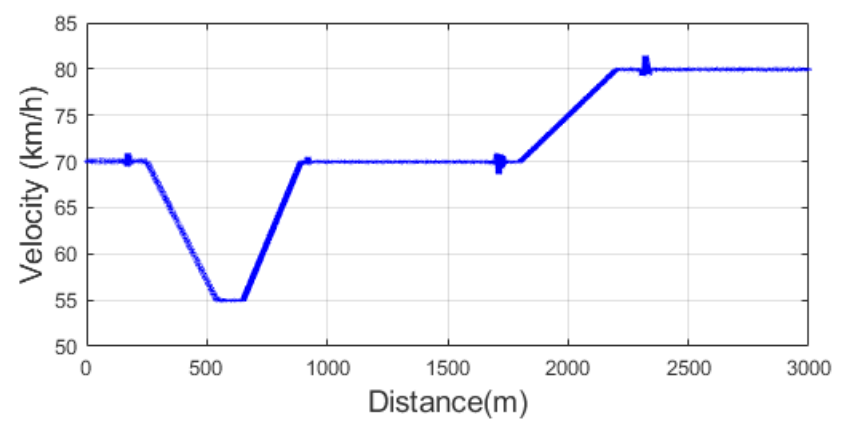

Fig. 4 Velocity of the simulated vehicles

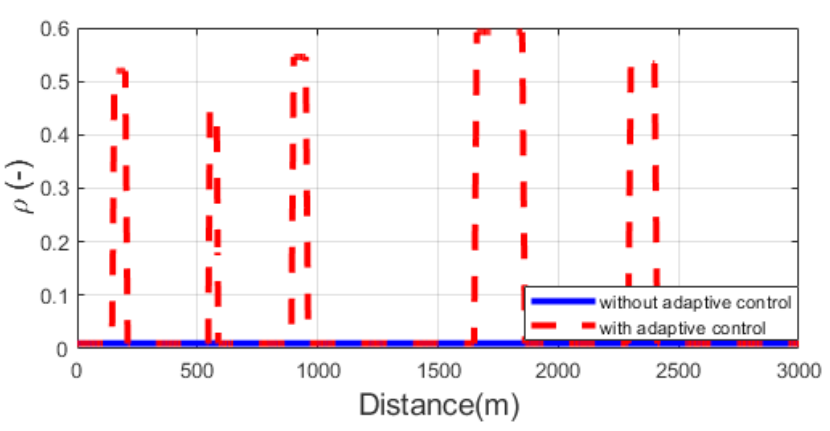

Fig. 5 Designed scheduling variables

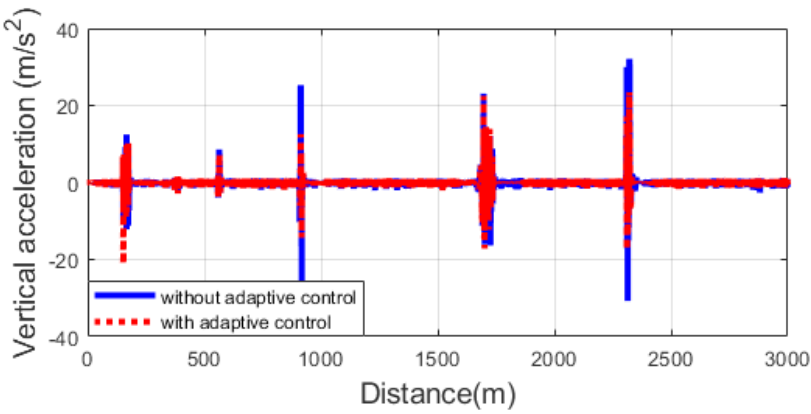

(a)

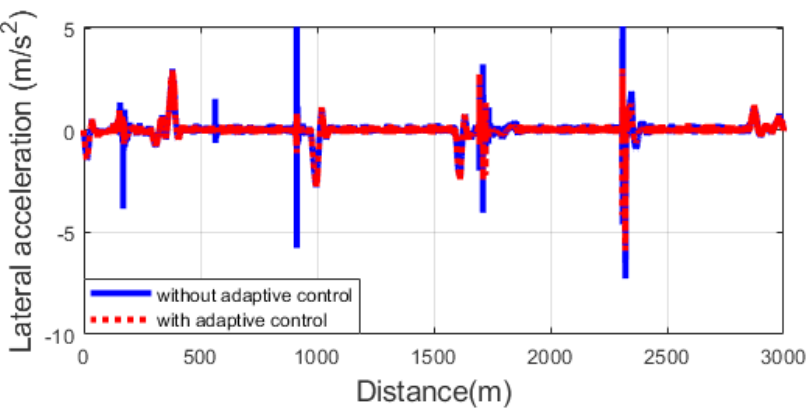

(b)

Fig. 6 (a) Vertical acceleration (b) Lateral acceleration

Moreover, tire deformation depicted in Fig. 7 and suspension deflection shown in Fig. 8 has also slightly reduced, thus road holding and stability has also been improved with the introduced method.

Note, that with the smoother operation of the vehicle suspension, control forces has also been reduced with the proposed adaptive system. For an example, control forces of the front-right suspension are depicted in Fig. 9 for the two simulated cases, showing reduced values for the cloud-based semi-active controller.

\section{Conclusion}

The paper proposed a novel cloud-based adaptive semi-active suspension system, adapting the controller's performance specifications to upcoming road conditions. The road quality information along with vehicle dynamic signals had been gathered and sent to the cloud database by 


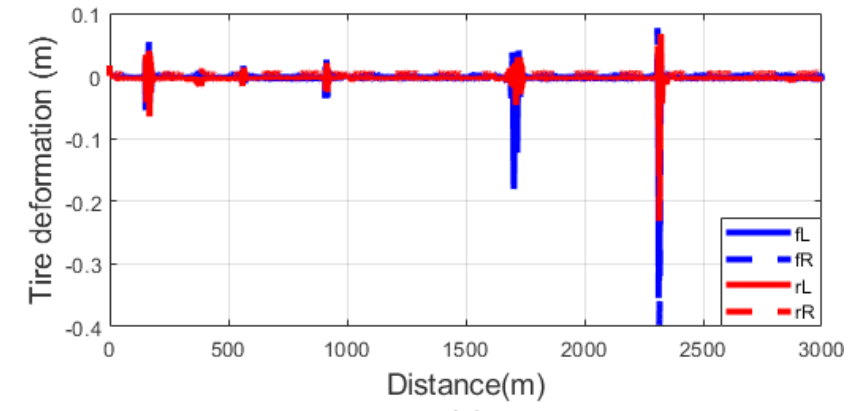

(a)

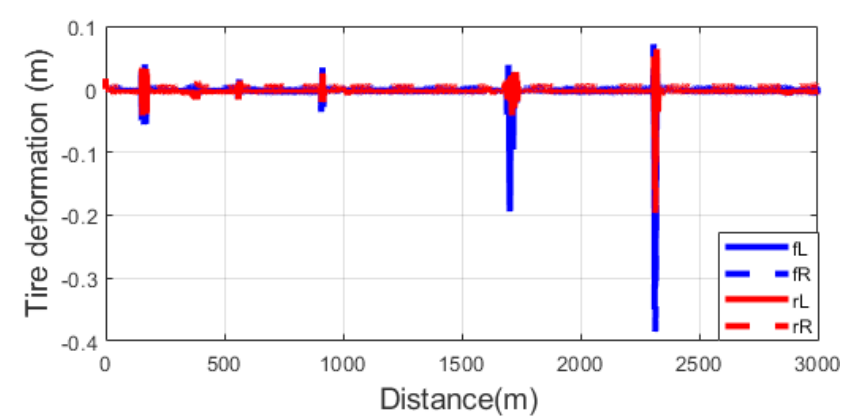

(b)

Fig. 7 Tire deformation; (a) without adaptive control; (b) with adaptive control

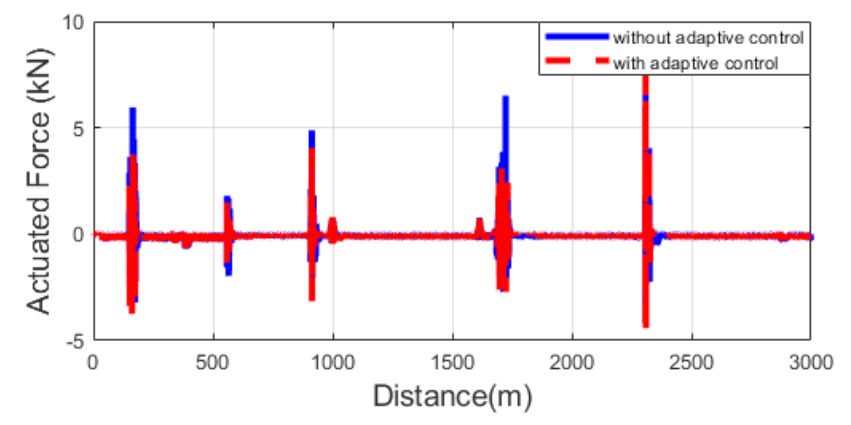

Fig. 8 Actuated force

previous journeys of the vehicles, where the corresponding scheduling variables for given road sections and vehicle velocities have been defined. By this means, the vehicle is able to adapt its suspension control system in coherence with oncoming road conditions and current velocity, which improves both ride quality and stability of the vehicle. The control reconfiguration has been designed founded on the LPV framework, while the operation of the proposed

\section{References}

Basargan, H., Mihály, A., Gáspár, P., Sename, O. (2020a) "Integrated multi-criteria velocity and semi-active suspension control based on look-ahead road information", In: 28th Mediterranean Conference on Control and Automation, Saint-Raphaël, France, pp. 25-30. https://doi.org/10.1109/MED48518.2020.9182953

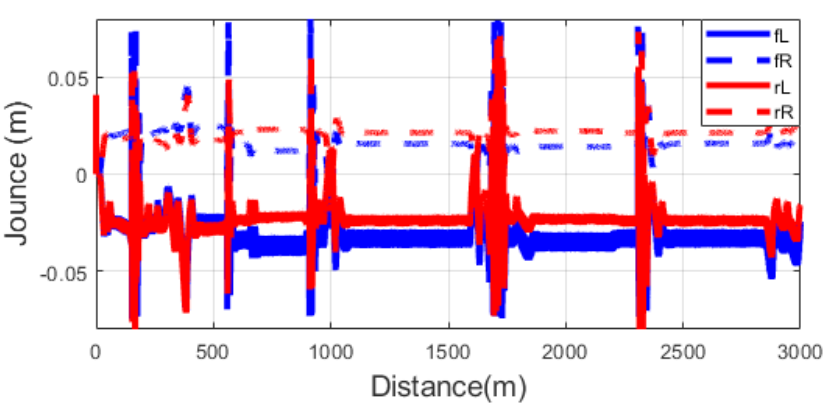

(a)

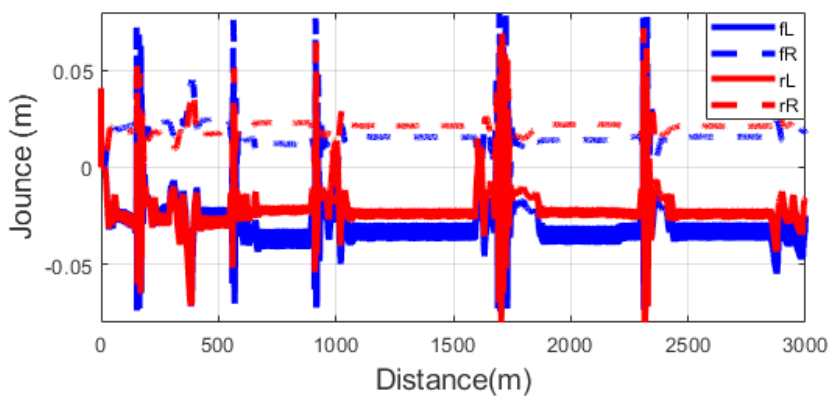

(b)

Fig. 9 Suspension deflection; (a) without adaptive control; (b) with adaptive control

method has been demonstrated in a real data simulation in TruckSim environment. The results show that both driving comfort and stability of the vehicle has been improved.

\section{Acknowledgement}

The research was supported by the Ministry for Innovation and Technology and the National Research, Development and Innovation Office within the framework of the National Lab for Autonomous Systems. This paper was partially supported by the National Research, Development and Innovation Office through the project "Integration of velocity and suspension control to enhance automated driving comfort in road vehicles" (NKFIH 2018-2.1.13-TÉT-FR). The research was also partially supported by the Hungarian Government and cofinanced by the European Social Fund through the project "Talent management in autonomous vehicle control technologies" (EFOP-3.6.3-VEKOP- 6-2017-00001).
Basargan, H., Mihály, A., Gáspár, P., Sename, O. (2020b) "Adaptive semi-active suspension control considering look-ahead road Information and irregularities", presented at 17th Mini Conference on Vehicle System Dynamics, Identification and Anomalies, Budapest, Hungary, November 102020. 
Basargan, H., Mihály, A., Gáspár, P., Sename, O. (2021) "Adaptive SemiActive Suspension and Cruise Control through LPV Technique", Applied Sciences, 11(1), Article number: 290

https://doi.org/10.3390/app11010290

Bokor, J., Balas, G. (2005) "Linear parameter varying systems: A geometric theory and applications", IFAC Proceedings Volumes, 38(1), pp. 12-22.

https://doi.org/10.3182/20050703-6-CZ-1902.00003

Guanetti, J., Borrelli, F. (2017) "Stochastic MPC for cloud-aided suspension control", In: 2017 IEEE 56th Annual Conference on Decision and Control (CDC), Melbourne, VIC, Australia, pp. 238-243. http://doi.org/10.1109/CDC.2017.8263672

Liu, C., Chen, L., Yang, X., Zhang, X., Yang, Y. (2019) "General theory of skyhook control and its application to semi-active suspension control strategy design", IEEE Access, 7, pp. 101552-101560. https://doi.org/10.1109/ACCESS.2019.2930567

Li, Z., Kolmanovsky, I., Atkins, E., Lu, J., Filev, D., Michelini, J. (2014) "Cloud aided semi-active suspension control", In: 2014 IEEE Symposium on Computational Intelligence in Vehicles and Transportation Systems (CIVTS), Orlando, FL, USA, pp. 76-83. https://doi.org/10.1109/CIVTS.2014.7009481

Li, Z., Kolmanovsky, I., Atkins, E., Lu, J., Filev, D. (2015) " $H_{\infty}$ Filtering for Cloud-Aided Semi-active Suspension with Delayed Road Information", IFAC-PapersOnLine, 48(12), pp. 275-280. https://doi.org/10.1016/j.ifacol.2015.09.390

Mihály, A., Kisari, Á., Gáspár, P., Németh, B. (2017) "Design of adaptive vehicle suspension using cloud-based road data", In: 2017 IEEE International Conference on Advanced Intelligent Mechatronics (AIM), Munich, Germany, pp. 529-534. http://doi.org/10.1109/AIM.2017.8014071

Moaaz, A. O., Ghazaly, N. M. (2019) "Semi-Active Suspension System Control Using Skyhook and Groundhook Controller", International Journal of Advanced Science and Technology, 28(18), pp. 424-433. [online] Available at: http://sersc.org/journals/index.php/IJAST/ article/view/2463 [Accessed: 08 December 2020]

Morato, M. M., Nguyen, M. Q., Sename, O., Dugard, L. (2019) Design of a fast real-time $L P V$ model predictive control system for semi-active suspension control of a full vehicle", Journal of the Franklin Institute, 356(3), pp. 1196-1224.

https://doi.org/10.1016/j.jfranklin.2018.11.016
Pham, T. P. (2020) "LPV observer and Fault-tolerant control of vehicle dynamics: application to an automotive semi-active suspension system", PhD dissertation, Université Grenoble Alpes. Available at: https://hal.univ-grenoble-alpes.fr/tel-02961042 [Accessed: 08 December 2020]

Rathai, K. M. M., Sename, O., Alamir, M. (2019a) "Reachability based Model Predictive Control for Semi-active Suspension System", In: 2019 Fifth Indian Control Conference (ICC), New Delhi, India, pp. $68-73$. https://doi.org/10.1109/INDIANCC.2019.8715601

Rathai, K. M. M., Alamir, M., Sename, O. (2019b) "Experimental implementation of model predictive control scheme for control of semi-active suspension system", IFAC-PapersOnLine, 52(5), pp. 261-266. https://doi.org/10.1016/j.ifacol.2019.09.042

Shimoya, N., Katsuyama, E. (2019) "A Study of Vehicle Ride Comfort using Triple Skyhook Control for Semi-active Suspension System", Transactions of Society of Automotive Engineers of Japan, 50(6), pp. 1631-1636. https://doi.org/10.11351/jsaeronbun.50.1631

Wu, F., Yang, X. H., Packard, A., Becker, G. (1995) "Induced L/sub 2/-norm control for LPV system with bounded parameter variation rates", In: Proceeding of 1995 American Control Conference - ACC'95, Seattle, WA, USA, pp. 2379-2383. https://doi.org/10.1109/ACC.1995.531398

Yu, S., Zhang, J., Xu, F., Chen, H. (2019) " $H_{\infty}$ Control of Semi-Active MR Damper Suspensions", In: 2019 12th Asian Control Conference (ASCC), Kitakyushu, Japan, pp. 337-342.

Ye, H., Zheng, L. (2019) "Comparative study of semi-active suspension based on LQR control and $\mathrm{H} 2 / \mathrm{H} \infty$ multi-objective control", In: 2019 Chinese Automation Congress (CAC), Hangzhou, China, pp. 3901-3906. https://doi.org/10.1109/CAC48633.2019.8996771

Zheng, X., Zhang, H., Yan, H., Yang, F., Wang, Z., Vlacic, L. (2019) "Active Full-Vehicle Suspension Control via Cloud-Aided Adaptive Backstepping Approach", IEEE Transactions on Cybernetics, 50(7), pp. 3113-3124.

http://doi.org/10.1109/TCYB.2019.2891960 